Pacific Journal of Mathematics

ON STRATIFIABLE SPACES 


\section{ON STRATIFIABLE SPACES}

\section{CARLOS J. R. Borges}

In the enclosed paper, it is shown that (a) the closed continuous image of a stratifiable space is stratifiable (b) the well-known extension theorem of Dugundji remains valid for stratifiable spaces (see Theorem 4.1, Pacific J. Math., 1 (1951), 353-367) (c) stratifiable spaces can be completely characterized in terms of continuous real-valued functions (d) the adjunction space of two stratifiable spaces is stratifiable (e) a topological space is stratifiable if and only if it is dominated by a collection of stratifiable subsets (f) a stratifiable space is metrizable if and only if it can be mapped to a metrizable space by a perfect map.

In [4], J. G. Ceder studied various classes of topological spaces, called $M_{i}$-spaces $(i=1,2,3)$, obtaining excellent results, but leaving questions of major importance without satisfactory solutions. Here we propose to solve, in full generality, two of the most important questions to which he gave partial solutions (see Theorems 3.2 and 7.6 in [4]), as well as obtain new results. ${ }^{1}$ We will thus establish that Ceder's $M_{3}$-spaces are important enough to deserve a better name and we propose to call them, henceforth, STRATIFIABLE spaces. Since we will exclusively work with stratifiable spaces, we now exhibit their definition.

Definition 1.1. A topological space $X$ is a stratifiable space if $X$ is $T_{1}$ and, to each open $U \subset X$, one can assign a sequence $\left\{U_{n}\right\}_{n=1}^{\infty}$ of open subsets of $X$ such that

(a) $U_{n}^{-} \subset U$,

(b) $U_{n=1}^{\infty} U_{n}=U$,

(c) $U_{n} \subset V_{n}$ whenever $U \subset V$.

For convenience, we will say that $\left\{U_{n}\right\}$ (more precisely, $\left\{U_{n}\right\}_{n=1}^{\infty}$ ) is a stratification of $U$ whenever the $U_{n}$ satisfy (a) and (b) of Definition 1.1. Similarly, we will say that the correspondence $U \rightarrow\left\{U_{n}\right\}$ is a stratification of $X$ whenever the $U_{n}$ satisfy (a), (b) and (c) of Definition 1.1. Certainly, we may suppose that any stratification $U \rightarrow\left\{U_{n}\right\}$ of $X$ is increasing, i.e. $U_{n} \subset U_{n+1}$ for each $n$ (if $U \rightarrow\left\{U_{n}\right\}$ is a stratification of $X$, then so is $U \rightarrow\left\{U_{n}^{\prime}\right\}$, where $\left.U_{n}^{\prime}=\bigcup_{i=1}^{n} U_{i}\right)$, a fact that will actually be used in $\S 4$. The same applies to stratifications of

Received November 23, 1964.

1 We express our sincere thanks to Professor E. A. Michael for his enthusiastic, helpful and encouraging supervision of this investigation. Nearly all our terminology is consistent with that used in Kelley [9], except that our spaces are always $T_{1}$. 
sets. ${ }^{2}$

For best appreciation of stratifiable spaces, we state some of the principal results of Ceder $((\mathrm{A})-(\mathrm{I}))$, our improvements of $(\mathrm{E})-(\mathrm{I})$, and some other results.

(A) The class of metrizable spaces is contained in the class of stratifiable spaces, and the latter class is contained in the class of perfectly paracompact spaces. ${ }^{3}$

(B) A stratifiable space is hereditarily stratifiable.

(C) The countable product of stratifiable spaces is stratifiable.

(D) In a stratifiable space $X$, the following are equivalent:

(a) $X$ is Lindelöf,

(b) $X$ is separable,

(c) $X$ satisfies the countable chain condition.

(E) If $A$ is a closed subset of a stratifiable space $X$, then the space $X / A$, obtained by identifying all points of $A$, is stratifiable.

(F) Dugundji's extension theorem (4.1 in [5]) is valid for stratifiable first countable spaces.

(G) If a topological space $X$ is a locally finite union of closed stratifiable subspaces, then $X$ is stratifiable.

(H) Every chunk-complex (see definition on p. 188 in [4]), and thus every $C W$-complex, is stratifiable. ${ }^{4}$

(I) A locally compact (more generally, absolute $G_{\delta}$ ) stratifiable space is metrizable.

Our improvements of $(\mathrm{E})$ - (I), where $\left(G^{\prime}, H^{\prime}\right)$ simultaneously generalizes (G) and $(\mathrm{H})$, are

$\left(\mathrm{E}^{\prime}\right)$ The closed continuous image of a stratifiable space is stratifiable $(\S 3)$.

$\left(\mathrm{F}^{\prime}\right)$ Dugundji's extension theorem is valid for stratifiable spaces $(\S 4)$.

$\left(\mathrm{G}^{\prime}, \mathrm{H}^{\prime}\right)$ A topological space, which is dominated (see Definition 7.1) by stratifiable subsets, is stratifiable $(\S 7)$.

$\left(\mathrm{I}^{\prime}\right)$ A stratifiable space is metrizable if and only if it can be mapped to some metrizable space by a perfect map (section 8 ). ${ }^{5}$

${ }^{2}$ Definition of 1.1 is clearly equivalent to Definition 1.3 in [4] (If $U \rightarrow\left\{U_{n}\right\}$ is a stratification of $X$, then $\mathscr{P}=\bigcup_{n=1}^{\infty} \mathscr{P}_{n}$ is $\sigma$-cushioned pair-base for $X$ if $\mathscr{P}_{n}=\left\{\left(U_{n}, U\right) \mid U\right.$ is an open subset of $X\}$. If $X$ has a $\sigma$-cushioned pair-base $\mathscr{B}=\mathrm{U}_{n=1}^{\infty} \mathscr{D}_{n}$, to each open $U \subset X$ assign the sequence $\left\{U_{n}\right\}_{n=1}^{\infty}$, where $\left.U_{n}=U\left\{P_{1} \in \mathscr{B}_{n} \mid P_{2} \subset U\right\}\right)$. Ceder proved the above equivalence in his dissertation (University of Washington, 1959).

${ }^{3}$ A topological space $X$ is said to be perfectly paracompact if it is paracompact and every closed subset of $X$ is a $G_{\delta}$ in $X$.

${ }^{4}$ Ceder actually showed that chunk-complexes are $M_{1}$ (Ceder showed that $M_{1} \mathrm{im}$ plies stratifiable, but it remains unknown whether the converse is true).

5 This actually strengthens (I), because Z. Frolik [6] proved that any paracompact absolute $G_{\delta}$-space can be mapped to a completely metrizable space by a perfect map, and conversely. 
Moreover, we show

$(\mathrm{J})$ The adjunction space $X \mathrm{U}_{f} Y$ of two stratifiable spaces $X$ and $Y$ is stratifiable $(\S 6)$.

We also characterize stratifiable spaces by continuous functions $(\S 5)$ and consider perfect inverse images of stratifiable spaces $(\S 8)$.

The above results show that stratifible spaces combine many of the desirable properties of metrizable spaces $\left((B),(C),(D),(G),\left(F^{\prime}\right)\right)$ and paracompact spaces $\left(\left(E^{\prime}\right),\left(G^{\prime}, H^{\prime}\right),(J)\right)$.

The following are some problems that remain unsolved:

1. If $X$ is compact Hausdorff and $Y$ is stratifiable, is the function space $Y^{X}$ (compact-open topology) again stratifiable? (It is not even known whether $Y^{X}$ is normal when $X$ is the unit interval and $Y$ is a simplicial $C W$-complex). We strongly suspect that the answer is "no".

2. Must a regular space, which is a continuous image of a separable stratifiable space, be stratifiable? (It is not even known whether a countable regular space must be stratifiable).

3. Is every separable stratifiable space a continuous image of a separable metrizable space?

For the reader's convenience, we observe that all sections, after $\S 2$, are independent of each other.

We end this section by stating a very crucial consequence of Definition 1.1, the proof of which appears in the next section:

To each pair $(A, U)$ of subsets of a stratifiable space, with $A$ closed, $U$ open and $A \subset U$, one can assign an open set $U_{A}$ satisfying

$$
A \subset U_{A} \subset\left(U_{A}\right)^{-} \subset U
$$

and

$$
U_{A} \subset V_{B} \text { whenever } U \subset V \text { and } A \subset B \text {. }
$$

2. Preliminary lemmas. Throughout this section, let $X$ be a stratifiable space.

Lemma 2.1. To each pair $(A, U)$ of subsets of $X$, with $A$ closed and $U$ open, we can assign an open set $U_{A} \subset U$ such that

(a) $U_{A} \subset V_{B}$ whenever $A \subset B$ and $U \subset V$,

(b) $A \cap U \subset U_{A} \subset\left(U_{A}\right)-\subset A \cup U$,

(b') $A \subset U_{A} \subset\left(U_{A}\right)^{-} \subset U$ whenever $A \subset U$. 
Proof. Let $U \rightarrow\left\{U_{n}\right\}$ be a stratification of $X$. To each pair $(A, U)$ of subsets of $X$, with $A$ closed and $U$ open, assign the set

$$
U_{A}=\bigcup_{n=1}^{\infty}\left(U_{n}-(X-A)_{n}^{-}\right) \text {. }
$$

Clearly, $U_{A}$ is open and $U_{A} \subset U$. Let us check the other requirements:

(a) Immediately, $U_{A} \subset V_{B}$ whenever $A \subset B$ and $U \subset V$.

(b) $A \cap U \subset U_{A}$ : Let $x \in A \cap U$; then $x \in U_{k}$, for some $k$, and $x \notin(X-A)$; thus $x \in U_{k}-(X-A)_{\bar{k}}^{-} \subset U_{A}$.

Also $\left(U_{A}\right) \subset A \cup U$ : If $x \notin A \cup U$, pick $n$ so that $x \in(X-A)_{n}$; then $(X-A)_{n} \cap\left(X-U_{n}^{-}\right)$is a neighborhood of $x$ disjoint from $U_{A}$.

( $\left.b^{\prime}\right)$ This is an immediate consequence of (b), since $A \subset U$ implies. $A \cap U=A$ and $A \cup U=U$.

The following lemma is needed in $\S 7$.

Lemma 2.2. Let $A$ be a closed subset of $X$. If $U \rightarrow\left\{U_{n}\right\}$ (U relatively open in $A$ ) is a stratification of $A$, then there exists $a$. stratification $V \rightarrow\left\{V_{n}\right\}$ ( $V$ open in $X$ ) of $X$ such that

(a) $V_{n} \cap A=(V \cap A)_{n}$
(b) $V_{n}^{-} \cap A=(V \cap A)_{n}^{-}$.

The preceding lemma is an immediate consequence of the following more detailed result, which is also needed in $\S 6$.

Lemma 2.3. Let $A$ be a closed subset of $X$. To each open $V \subset X$ and stratification $\left\{\alpha_{n}(V \cap A)\right\}$ of $V \cap A$ (relative to $A$ ) one can assign a stratification $\left\{\alpha_{n}(V)\right\}$ of $V$ such that

(a) $\alpha_{n}(V) \cap A=\alpha_{n}(V \cap A)$

(b) $\left[\alpha_{n}(V)\right]^{-} \cap A=\left[\alpha_{n}(V \cap A)\right]^{-}$,

(c) $\alpha_{n}(V) \subset \beta_{n}(W)$ whenever $\alpha_{n}(V \cap A) \subset \beta_{n}(W \cap A)$ and $V \subset W$.

Proof. Let $U \rightarrow\left\{U_{n}\right\}$ be a stratification of $X$. For each open $V \subset X$, each stratification $\left\{\alpha_{n}(V \cap A)\right\}$ of $V \cap A$ (relative to $A$ ), and all $n$, let

$$
\alpha_{n}(V)=(V-A)_{n} \cup\left[(V-A) \cup \alpha_{n}(V \cap A)\right]\left[\alpha_{n}(V \cap A)\right]^{-} .
$$

By Lemma 2.1 (b) and by the definition of $U_{A}$,

(1) $(V-A)_{n} \cup \alpha_{n}(V \cap A) \subset \alpha_{n}(V) \subset(V-A) \cup \alpha_{n}(V \cap A)$,

(2) $\quad \alpha_{n}(V \cap A) \subset\left[\alpha_{n}(V)\right]^{-} \subset(V-A) \cup\left[\alpha_{n}(V \cap A)\right]^{-} \subset V$.

Now all requirements of this lemma easily follow from (1), (2), and the fact that $V=\bigcup_{n=1}^{\infty} \alpha_{n}(V)$ (since $\bigcup_{n=1}^{\infty} \alpha_{n}(V \cap A)=V \cap A$ and $\left.\mathrm{U}_{n=1}^{\infty}(V-A)_{n}=V-A\right)$. 


\section{Closed continuous maps.}

TheOREM 3.1. The closed continuous image of a stratifiable space is stratifiable.

Proof. Let $f: X \rightarrow Y$ be a closed continuous map from the stratifiable space $X$ onto $Y$, and let $U \rightarrow\left\{U_{n}\right\}$ be a stratification of $X$. For each open subset $V$ of $Y^{\prime}$ let

$$
\begin{aligned}
T_{n} & =\left[f^{-1}(V)\right]_{n}, & S_{n} & =f^{-1}\left(f\left(T_{n}^{-}\right)\right), \\
Q_{n} & =\left[f^{-1}(V)\right] S_{n}, & V_{n} & =\left[f\left(Q_{n}\right)\right]^{0} .
\end{aligned}
$$

Then

(a) $V_{n}$ is a neighborhood of $f\left(T_{n}^{-}\right)$: By Lemma $2.1\left(\mathrm{~b}^{\prime}\right), Q_{n}$ is a neighborhood of the (inverse) set $S_{n}$; since $f$ is closed, $f\left(Q_{n}\right)$ is a neighborhood of $f\left(T_{n}^{-}\right)$.

(b) $\quad V_{n}^{-} \subset V: V_{n}^{-} \subset\left[f\left(Q_{n}\right)\right]^{-}=f\left(Q_{n}^{-}\right) \subset V$, since $f$ is closed, and $Q_{n}^{-} \subset f^{-1}(V)$ by Lemma $2.1\left(b^{\prime}\right)$.

(c) $\bigcup_{n=1}^{\infty} V_{n}=V$ : By (a) and the definitions, $V \supset V_{n} \supset f\left(T_{n}\right)$ and $\bigcup_{n=1}^{\infty} f\left(T_{n}\right)=f\left(\bigcup_{n=1}^{\infty} T_{n}\right)=V$.

Clearly, if $V$ and $W$ are open subsets of $Y$, and $V \subset W$, then $V_{n} \subset W_{n}$, since all preceding operations are order-preserving. Thus $V \rightarrow\left\{V_{n}\right\}$ is a stratification of $Y$.

In [4], Ceder defined the class of Nagata spaces (see Definition 3.1 in [4]) which he showed to be the same as the class of stratifiable first countable spaces. We have thus established:

Corollary 3.2. The closed continuous image of a Nagata space is a Nagata space if and only if it is first countable.

Remark 3.3. Morita and Hanai [13], and Stone [15] imdependently proved that if $f: X \rightarrow Y$ is a closed continuous map from the metrizable space $X$ onto the $T_{1}$-topological space $Y$, then the following are equivalent:

(a) $Y$ is metrizable,

(b) $Y$ is first countable,

(c) bdry $f^{-1}(y)$ is compact for each $y \in Y$.

Corollary 3.2 shows that part of above result remains valid for Nagata spaces. However, it is worthwhile noting that a complete duplicate of Stone's result cannot be obtained for Nagata spaces, since the subspace $S=[0,1] \times\{0\}$ of the Nagata space $X$, constructed in Example 9.2 in [4] $(X=\{(x, y) \mid x$ and $y$ are real and $y \geqq 0\}$; the topology on $X$ has a base consisting of disks missing the $x$-axis and sets of the form $U_{n}(p)=\{(x, y)|| s-p \mid<1 / n$ and $y$ lies below the 
graph of $\left.\left.(x-p)^{2}+(y-n)^{2}=n^{2}\right\} \cup\{p\}\right)$, can be shown to be compact, but the quotient space obtained by identifying all points of $S$ is a nonfirst countable closed continuous image of $X$.

Another application of Theorem 3.1 is to prove that Theorem 2 of Stone [15], which he proved for metrizable spaces, remains valid for stratifiable spaces. We first need the following lemma, which Stone also proved for metrizable spaces.

LEMma 3.4. Let $f: X \rightarrow Y$ be a monotone quotient map from the locally peripherally compact ${ }^{6}$ space $X$ onto the Hausdorff space $Y$ such that, for each $p \in Y, b d r y f^{-1}(p)$ is compact. Then $f$ is closed and $Y$ is locally peripherally compact.

Proof. For each $p \in Y$, let $F_{p}=f^{-1}(p)$. Given any open set $U \supset F_{p}$, we must find an open set $R$, with $F_{p} \subset R \subset U$, such that $F_{q} \subset U$ whenever $F_{q}$ intersects $R$.

Cover bdry $F_{p}$ by a finite number of open sets $U_{1}, \cdots, U_{m}$ such that $U_{i} \subset U$ and bdry $U_{i}$ is compact $(1 \leqq i \leqq m)$. Let $V=\left(\bigcup_{i=1}^{m} U_{i}\right) \cup\left(F_{p}\right)^{0}$. Then $F_{p} \subset V \subset U$ and bdry $V$ is compact.

Since $f($ bdry $V)$ is compact, and hence closed in $Y$, the set $W=$ $f^{-1}[Y-f$ (bdry) $]$ is an open neighborhood of $F_{p}$. If now $F_{q}$ intersects $R=W \cap V$, then $F_{q} \subset W$, and hence $F_{q} \cap$ bdry $V=\phi$. Thus, since $F_{q}$ is connected, $F_{q} \subset V$.

To prove that $Y$ is locally peripherally compact, we proceed as in proof of Theorem 2 of Stone [15].

CoRollary 3.5. Let $f: X \rightarrow Y$ be a monotone quotient map from the locally peripherally compact stratifiable space $X$ onto the Hausdorff space $Y$. Then $Y$ is a locally peripherally compact stratifiable space.

4. Dugundji's extension theorem. After a couple of preliminaries, we will show that the well-known extension theorem of Dugundji for metric spaces (Theorem 4.1 in [5]) remains valid for stratifiable spaces.

Definition 4.1. For each open subset $U$ of $X$ and $x \in U$, let $n(U, x)$ be the smallest integer $n$ such that $x \in U_{n}$, and let

$$
U_{x}=U_{n(U, x)}-(X-\{x\})_{n(U, x)}^{-} .
$$

${ }^{6}$ A topological space $X$ is locally peripherally compact if, for each $x \in X$ and neighborhood $U$ of $x$, there exists a neighborhood $V$ of $x$ such that $V \subset U$ and bdry $V$ is compact. For any set $S$, "bdry $S$ " means "the boundary of $S$. " 
Observe that $U_{x}$ and $U_{\{x\}}(\S 2)$ are not necessarily the same sets.

Lemma 4.2. For $U, V$ open subsets of $X, x \in U$ and $y \in V$, we have the following:

(i) $U_{x}$ is an open neighborhood of $x$,

(ii) $U_{x} \cap V_{y} \neq \phi$ and $n(U, x) \leqq n(V, y)$ implies $y \in U$,

(iii) $U_{x} \cap V_{y} \neq \phi$ implies $x \in V$ or $y \in U$.

Proof. Clearly, $U_{x}$ is an open neighborhood of $x$, by the definition of $U_{x}$; also (iii) is an immediate consequence of (ii), since either $n(V, y) \leqq n(U, x)$ or $n(U, x) \leqq n(V, y)$.

Therefore, we proceed to prove (ii): Assume $y \notin U$. Then

$$
(X-\{y\})_{n} \supset U_{n}
$$

for each $n$; therefore

$$
(X-\{y\})_{n(V, y)} \supset(X-\{y\})_{n(U, x)} \supset U_{n(U, x)} .
$$

Consequently, by Definition 4.1, $U_{x} \cap V_{y}=\phi$, a contradiction. Thus $y \in U$.

THEOREM 4.3. Let $X$ be a stratifiable space, $A$ a closed subset of $X, E$ a locally convex linear topological space, $C(X, E)$ the linear space of continuous functions from $X$ into $E$, and similarly for $C(A, E)$. Then there exists a mapping

$$
\phi: C(A, E) \rightarrow C(X, E)
$$

satisfying the following conditions:

(a) $\phi(f)$ is an extension of $f$ for every $f \in C(A, E)$,

(b) the range of $\phi(f)$ is contained in the convex hull of the range of $f$, for every $f \in C(A, E)$,

(c) $\dot{\phi}$ is a linear transformation. ${ }^{7}$

Proof. Let $W=X-A$, and let $W^{\prime}=\left\{x \in W \mid x \in U_{y}\right.$ for some $y \in A$ and open $U$ containing $y\}$. For each $x \in W^{\prime}$, let

$$
m(x)=\max \left\{n(U, y) \mid y \in A \text { and } x \in U_{y}\right\} .
$$

Note that $m(x)>\infty$ and, in fact, $m(x)<n(W, x)$ : If not, there exists $y \in A$ and open neighborhood $U$ of $y$, such that $x \in U_{y}$ (thus

${ }^{7}$ In [1] and [10], it is shown that Theorem 4.3 is not valid for compact Hausdorff (hence paracompact) spaces. It seems to be unknown whether Theorem 4.3 is valid for perfectly paracompact spaces. 
$\left.W_{x} \cap U_{y} \neq \phi\right)$ and $n(U, y) \geqq n(W, x)$; hence $y \in W$, by Lemma 4.2 (ii), which is impossible.

Using the paracompactness of $W$, let $\mathscr{V}$ be an open locally finite (with respect to $W$ ) refinement of $\left\{W_{x} \mid x \in W\right\}$. For each $V \in \mathscr{V}$, pick $x_{V} \in W$ such that $V \subset W_{x_{V}}$. If $x_{V} \in W^{\prime}$, pick $a_{V} \in A$ and open $S_{V}$ containing $a_{V}$ such that $x_{V} \in\left(S_{V}\right)_{a_{V}}$ and $n\left(S_{V}, a_{V}\right)=m\left(x_{V}\right)$; if $x_{V} \notin W^{\prime}$, let $a_{V}$ be the fixed point $a_{0} \in A$.

Let $\left\{p_{V} \mid V \in \mathscr{Y}\right\}$ be a partition of unity subordinated to $\mathscr{Y}$, and define $g: X \rightarrow E$ by

$$
g(x)=f(x)
$$

if $x \in A$,

and

$$
g(x)=\Sigma_{V \in \mathscr{N}}(x) f\left(a_{V}\right) \quad \text { if } x \in W .
$$

Then, clearly, $g(X) \subset$ convex hull of $f(A)$ and $g$ is continuous on $W$.

To show that $g$ is continuous at $b \in A$, let 0 be any open subset of $E$ containing $f(b)$. By the local convexity of $E$, there exists a convex open subset $K$ of $E$ such that $f(b) \subset K \subset 0$ and, by the continuity of $f$, there exists an open neighborhood $N$ of $b$ such that $f(A \cap N) \subset K \subset 0$.

Now we show that $g\left(\left(N_{b}\right)_{b}\right) \subset 0$ : If $x \in\left(N_{b}\right)_{b} \cap A \subset N \cap A$ then $g(x)=f(x) \in 0$. Let $x \in\left(N_{b}\right)_{b}-A$. Consider any $V \in \mathscr{Y}^{-}$with $x \in V$. Since $b \notin W_{x_{V}}$ and $x \in\left(N_{b}\right)_{b} \cap W_{x_{V}}$, we get that $x_{V} \in N_{b}$, by Lemma 4.2 (iii); hence $x_{V} \in W^{\prime}$ and $n(N, b) \leqq m\left(x_{V}\right)=n\left(S_{V}, a_{V}\right)$. By Lemma 4.2 (ii), $a_{V} \in N$, since $x_{V} \in N_{b} \cap\left(S_{V}\right)_{a_{V}}$. Consequently $f\left(a_{V}\right) \in K$ and, by the convexity of $K, g(x) \in K \subset 0$. Hence $g\left(\left(N_{b}\right)_{b}\right) \subset 0$, establishing the continuity of $g$ on $A$.

To complete the proof, we simply let $\phi(f)=g$.

In [10] (Theorem 7.1), it is proved that, for any metrizable space $X, \phi$ can be chosen to be continuous whenever both $C(A, E)$ and $C(X, E)$ have the compact-open topology, the topology of pointwise convergence, or the topology of uniform convergence. Let us show that this remains valid for stratifiable spaces, using the function $\phi$ constructed in the proof of our Theorem 4.3 (We will need to assume that for each $V \in \mathscr{V}, V^{-} \subset W_{x_{V}}$, where $V^{-}$denotes the closure of $V$ with respect to $W$, which is easily done). As in the proof of Lemma 7.2 in [10], it suffices to show that the function $u: X \rightarrow 2^{A}$, defined by

$$
u(x)=\{x\}
$$




$$
u(x)=\left\{a_{V} \mid V \in \mathscr{Y}^{-} \text {and } x \in V^{-}\right\} \quad \text { if } x \in W,
$$

is upper semi-continuous ${ }^{8}$ at points of $A$ :

Let $p \in A$ and $U$ be an open subset of $X$ containing $u(p)=\{p\}$. We show that, for each $x \in\left(U_{p}\right)_{p}, u(x) \subset U$ : If $x \in A \cap\left(U_{p}\right)_{p}$ then $u(x)=\{x\} \subset U$. If $x \in\left(U_{p}\right)_{p}-A$, consider any $V \in \mathscr{Y}$ with $x \in V^{-}$; since $p \notin W_{x_{V}}$ and $x \in\left(U_{p}\right)_{p} \cap W_{x_{V}}$, we get that $x_{V} \in U_{p}$, by Lemma 4.2 (ii); hence $x_{V} \in W^{\prime}$ and $n(U, p) \leqq m\left(x_{V}\right)=n\left(S_{V}, a_{V}\right)$. By Lemma 4.2 (iii), $a_{V} \in U$, since $x_{V} \in U_{p} \cap\left(S_{V}\right)_{a_{V}}$. Hence

$$
\left\{a_{V} \mid V \in \mathscr{Y} \text { and } x \in V^{-}\right\} \subset U
$$

and thus $u(x) \subset U$, completing the proof.

5. Characterization by continuous functions, Throughout this section, let $I$ be the closed unit interval.

THEOREM 5.1. Let $X$ be a stratifiable space. To each pair $(A, U)$ of subsets of $X$, with $A$ closed, $U$ open, and $A \subset U$, one can assign $a$ continuous function $f_{U, A}: X \rightarrow I$ such that $f_{U, A}(A)=0, f_{U,{ }_{A}}(X-U)=1$, and $f_{U, A} \geqq f_{V, B}$ whenever $A \subset B$ and $U \subset V$.

Proof. It suffices to reproduce Urysohn's lemma (Lemma 4, p. 115, [9]), using Lemma 2.1 (a) and $\left(b^{\prime}\right)$. Thus we will simply display the first steps of the required induction. We let

$$
\begin{gathered}
M(0)=U_{A} \\
M\left(\frac{1}{2}\right)=[M(1)]_{[M(0)]^{-}} \\
M\left(\frac{1}{4}\right)=\left[M\left(\frac{1}{2}\right)\right]_{[M(0)]^{-}} M\left(\frac{3}{4}\right)=[M(1)]_{[M(1 / 2)]^{-}} .
\end{gathered}
$$

Continuing in this fashion, we define $M(r)$ for all dyadic rationals in $I$ such that

$$
r_{1}<r_{2} \text { implies }\left[M\left(r_{1}\right)\right]^{-} \subset M\left(r_{2}\right) \text {. }
$$

Also, let $M(r)=X$ for $r>1$.

Now we define the function $f_{U, A}$ by

$$
f_{U, A}(x)=\inf \{r \mid x \in M(r) \text { and } r>0\}
$$

${ }^{8}$ If $X$ and $Y$ are topological spaces, a function $u: X \rightarrow 2^{Y}$ is upper semi-continuous at a point $x \in X$ if, for every open subset $U$ of $Y$ containing $u(x)$, there exists a neighborhood $W$ of $x$ in $X$ such that $u\left(x^{\prime}\right) \subset U$ for every $x^{\prime} \in W$; $u$ is called upper semicontinuous if it is upper semi-continuous at every $x \in X$. 
for all $x \in X$. By Lemma 3 (p. 114) in [9], $f_{V, A}$ is continuous. Clearly, $f_{V, A}(A)=0$ and $f_{U, A}(X-U)=1$. Finally, Lemma 2.1 (a) and our definitions (of the sets $M(r)$ ) imply that $f_{U, A} \geqq f_{V, B}$ whenever $A \subset B$ and $U \subset V$.

TheOREM 5.2. A topological space $X$ is stratifiable if and only if to each open $U \subset X$ one can assign a continuous function $f_{U}: X \rightarrow I$, such that $f_{U}^{-1}(0)=X-U$, and $f_{U} \leqq f_{V}$ whenever $U \subset V$.

Proof. To prove the "if" assertion, assume that to each open $U \subset X$ one can assign a continuous function $f_{U}: X \rightarrow I$ such that $f_{U}^{-1}(0)=X-U$, and $f_{U} \leqq f_{V}$ whenever $U \subset V$. For each open $U \subset X$ and all $n$, let

$$
U_{n}=X-f_{\bar{U}}^{-1}\left(\left[0, \frac{1}{n}\right]\right) .
$$

It is easily checked that $U \rightarrow\left\{U_{n}\right\}$ is a stratification of

$$
\begin{gathered}
X\left(\bigcup_{n=1}^{\infty} U_{n}=X-\bigcap_{n=1}^{\infty} f_{U}^{-1}\left(\left[0, \frac{1}{n}\right]\right)=U ;\right. \\
U_{n}^{-} \subset X-f_{U}^{-1}\left(\left[0, \frac{1}{n}\right]\right) \subset U ;
\end{gathered}
$$

$U \subset V$ implies $U_{n} \subset V_{n}$, since $f_{U} \leqq f_{V}$ ).

To prove the "only if" assertion, let $X$ be a stratifiable space and let $f_{U, A}$ be as in Theorem 5.1. Let

$$
f_{U}=\sum_{n=1}^{\infty} 2^{-n} f_{X-U_{n}^{-}, X-U} .
$$

It is well-known that $f_{U}$ is continuous and, clearly, $f_{U}^{-1}(0)=X-U$. Finally, one easily sees that $f_{U} \leqq f_{V}$ whenever $U \subset V$.

6. Adjunction spaces. The concept of adjunction space was first introduced by J. H. C. Whitehead, and later used by Hanner [7] in connection with extension and retraction properties of topological spaces.

Definition 6.1. Let $X$ and $Y$ be topological spaces, $A$ a closed subset of $X$ and $f: A \rightarrow Y$ a continuous function. Let $X \cup Y$ denote the topological disjoint union of $X$ and $Y$, and let $Z$ be the quotient space which we get from identifying each $x \in A$ with $f(x) \in Y$. Then $Z$ (commonly denoted by $X \bigcup_{f} Y$ ) is called the adjunction space of $X$ and $Y$ (see [8]). 
THeorem 6.2. Let $X$ and $Y$ be stratifiable spaces, $A$ a closed subset of $X$ and $f: A \rightarrow Y$ a continuous function. Then $X \cup_{f} Y$ is stratifiable.

Proof. Let $Z=X \bigcup_{f} Y$ and let $h: X \rightarrow Z, k: Y \rightarrow Z$ be the natural projections. As indicated by Hanner [7], $0 \subset Z$ is open (closed) if and only if $h^{-1}(0)$ and $k^{-1}(0)$ are open (closed); furthermore, $k$ and $h \mid X-A$ are homeomorphisms into. Note that for any subset $B$ of $Z$,

$$
f^{-1} k^{-1}(B)=h^{-1}(B) \cap A .
$$

For convenience, for any open subset 0 of $Z$, we let

$$
\begin{gathered}
O_{Y}=k^{-1}(0), \quad O_{X}=h^{-1}(0), \\
O_{A}=O_{X} \cap A=f^{-1}\left(O_{Y}\right) .
\end{gathered}
$$

Let $V \rightarrow\left\{V_{n}\right\}$ be a stratification of $Y$. Then, letting $\left(O_{Y}\right)_{n}=O_{Y, n}$ and $f^{-1}\left(O_{Y, n}\right)=O_{A, n}$, we see that $\left\{O_{A, n}\right\}$ becomes a stratification of $O_{A}$, for each open $0 \subset Z$. By Lemma 2.3, for each open $0 \subset Z$ there exists a stratification $\left\{O_{X, n}\right\}$ of $O_{X}$, satisfying

(a) $O_{X, n} \cap A=O_{A, n}$,

(b) $O_{\bar{x}, n}^{-} \cap A=O_{A}^{-}, n$,

(c) $0 \subset O^{\prime}$ implies $O_{x, n} \subset O_{X, n}^{\prime}$ : If $0 \subset O^{\prime}$, then $O_{A, n} \subset O_{A, n}^{\prime}$ (since $\left.O_{Y, n} \subset O_{Y, n}^{\prime}\right)$ and $O_{X} \subset O_{X}^{\prime}$, and hence $O_{X, n} \subset O_{X, n}^{\prime}$ by Lemma 2.3 (c). Now we let

$$
O_{n}=h\left(O_{X, n}\right) \cup k\left(O_{Y, n}\right) \text {. }
$$

To prove that $0 \rightarrow\left\{O_{n}\right\}$ is a stratification of $Z$, we will repeatedly use that

$$
\begin{aligned}
h^{-1} \circ k & =f^{-1}, \\
h^{-1} h(S) & =S \cup f^{-1} f(S \cap A) \\
k^{-1} h(S) & =f(S \cap A)
\end{aligned}
$$$$
\text { if } S \subset X \text {, }
$$$$
\text { if } S \subset X \text {. }
$$

We now show

(1) $O_{n}$ is an open subset of $Z$ : It suffices to show that $h^{-1}\left(O_{n}\right)$ and $k^{-1}\left(O_{n}\right)$ are open. But, using (a),

$$
\begin{aligned}
h^{-1}\left(O_{n}\right) & =h^{-1} h\left(O_{X, n}\right) \cup h^{-1} k\left(O_{Y, n}\right) \\
& =O_{X, n} \cup f^{-1} f\left(O_{X, n} \cap A\right) \cup f^{-1}\left(O_{Y, n}\right) \\
& =O_{X, n} \cup f^{-1} f\left(O_{A, n}\right) \cup O_{A, n} \\
& =O_{X, n} \cup O_{A, n} \\
& =O_{X, n} .
\end{aligned}
$$

Similarly, 


$$
\begin{aligned}
k^{-1}\left(O_{n}\right) & =k^{-1} h\left(O_{X, n}\right) \cup k^{-1} k\left(O_{Y, n}\right) \\
& =f\left(O_{X, n} \cap A\right) \cup O_{Y, n} \\
& =f\left(O_{A, n}\right) \cup O_{Y, n} \\
& =O_{Y, n} .
\end{aligned}
$$

(2) $O_{n}^{-} \subset 0$ : Let $C=h\left(O_{\bar{x}, n}^{-}\right) \cup k\left(O_{\bar{Y}, n}^{-}\right)$; it follows that $O_{n} \subset C \subset 0$ and thus it suffices to show that $C$ is closed (i.e. $h^{-1}(C)$ and $k^{-1}(C)$ are closed). But, using (b),

$$
\begin{aligned}
h^{-1}(C) & =h^{-1} h\left(O_{\bar{X}, n}^{-}\right) \cup h^{-1} k\left(O_{\bar{Y}, n}^{-}\right) \\
& =O_{\bar{X}, n}^{-} \cup f^{-1} f\left(O_{\bar{X}, n}^{-} \cap A\right) \cup f^{-1}\left(O_{\bar{Y}, n}^{-}\right) \\
& =O_{\bar{X}, n}^{-} \cup f^{-1}\left(f\left(O_{A}^{-}, n\right) \cup O_{\bar{Y}, n}^{-}\right) \\
& =O_{\bar{X}, n}^{-} \cup f^{-1}\left(O_{\bar{Y}, n}^{-}\right) .
\end{aligned}
$$

Similarly,

$$
\begin{aligned}
k^{-1}(C) & =k^{-1} h\left(O_{\bar{X}, n}^{-}\right) \cup k^{-1} k\left(O_{\bar{Y}, n}^{-}\right) \\
& =f\left(O_{\bar{Y}, n}^{-} \cap A\right) \cup O_{\bar{Y}, n}^{-} \\
& =f\left(O_{A}^{-}, n\right) \cup O_{\bar{Y}, n}^{-} \\
& =O_{\bar{Y}, n}^{-} .
\end{aligned}
$$

(3) $\bigcup_{n=1}^{\infty} O_{n}=" 0: \bigcup_{n=1}^{\infty} O_{n}=\bigcup_{n=1}^{\infty}\left[h\left(O_{X, n}\right) \cup k\left(O_{Y, n}\right)\right]$

$$
=h\left(\bigcup_{n=1}^{\infty} O_{X, n}\right) \cup k\left(\bigcup_{n=1}^{\infty} O_{Y, n}\right)=h\left(O_{X}\right) \cup k\left(O_{Y}\right)=0 .
$$

(4) If $0, O^{\prime}$ are open subsets of $Z$ and $0 \subset O^{\prime}$, then $O_{n} \subset O_{n}^{\prime}$ : immediate from (c) and the fact that $0 \subset O^{\prime}$ implies $O_{Y, n} \subset O_{Y, n}^{\prime}$.

Consequently, from (1)-(4), $0 \rightarrow\left\{O_{n}\right\}(0$ open in $Z)$ is a stratification of $Z$.

CoRollary 6.3. Let $Y$ be a stratifiable space. Then $Y$ is an $A R$ (stratifiable), resp. ANR (stratifiable), if and only if $Y$ is an $A E$ (stratifiable), resp. ANE (stratifiable). ${ }^{9}$

Proof. This follows from Theorem 6.2, using the same method of proof of Theorem 8.1 in [7].

7. Spaces dominated by stratifiable subsets. We start by reproducing Definition 8.1 in [11].

DEFINITION 7.1. Let $X$ be a topological space, and a collection of

${ }^{9} A R=$ absolute retract, $A N R=$ absolute neighborhood retract, $A E=$ absolute extensor, $A N E=$ absolute neighborhood extensor. 
closed subsets of $X$. Then $\mathscr{B}$ dominates $X$ if, whenever $A \subset X$ has a closed intersection with every element of some subcollection $\mathscr{B}_{1}$ of $\mathscr{B}$ which covers $A$, then $A$ is closed.

As examples of covers of a topological space $X$ which dominate $X$, we mention the following:

(1) locally finite closed covers of $X$,

(2) the family of finite subcomplexes of a $C W$-complex (more generally, the family of chunks of a chunk-complex).

In [11], it is then shown that a topological space is paracompact if and only if it is dominated by a collection of paracompact spaces (this result was also proved by K. Morita). We prove the following:

THEOREM 7.2. A topological space is stratifiable if and only if it is dominated by a collection of stratifiable subsets.

Proof. The "only if" assertion is obvious. Let us, therefore, prove the "if" assertion.

We begin by noting that the special case of two closed sets was already obtained by Ceder (Lemma 2.7 in [4]), a fact that will be used later.

Now let $\mathscr{B}$ be a dominating collection of stratifiable subsets of $X$. Consider the class $G$ of all pairs of the form $\left(\mathscr{C}_{\alpha}, S_{\alpha}\right)$, where $\mathscr{C}_{\alpha} \subset \mathscr{B}$, and $S_{\alpha}$ is a stratification of $C_{\alpha}=\mathrm{U} \mathscr{C}_{\alpha}$ which will be denoted by $V \rightarrow\left\{V_{\alpha, n}\right\} \quad\left(V\right.$ relatively open in $C_{\alpha}$ ). (Throughout this proof, $\bigcup \mathscr{C}_{\gamma}$ will be denoted by $C_{\gamma}$ for any $\mathscr{C}_{\gamma} \subset \mathscr{B}$ ). We partially order $G$ by letting $\left(\mathscr{C}_{\alpha}, S_{\alpha}\right) \leqq\left(\mathscr{C}_{\beta}, S_{\beta}\right)$ whenever $\mathscr{C}_{\alpha} \subset \mathscr{C}_{\beta}$ and, for each relatively open $U$ in $C_{\beta}$,

(a) $U_{\beta, n} \cap C_{\alpha}=\left(U \cap C_{\alpha}\right)_{\beta, n}$,

(b) $\quad U_{\beta, n}^{-} \cap C_{\alpha}=\left(U \cap C_{\alpha}\right)_{\bar{\beta}, n}$

for all $n$.

We now show that any simply ordered subfamily $\left\{\mathscr{C}_{\alpha}, S_{\alpha}\right\}_{\alpha} \in_{A}$ of $G$ has an upper bound $\left(\mathscr{C}_{\beta}, S_{\beta}\right)$. Let $\mathscr{C}_{\beta}=\bigcup_{\alpha \in_{A}} \mathscr{C}_{\alpha}$. For each relatively open $U$ in $C_{\beta}$, and all $n$, let

$$
U_{\beta, n}=\bigcup_{\alpha \in A}\left(U \cap C_{\alpha}\right)_{\alpha, n},
$$

and let us show that $U \rightarrow\left\{U_{\beta, n}\right\}$ is a stratification $S_{\beta}$ on $C_{\beta}$ which satisfies (a) and (b) for every $\alpha \in A$.

Clearly $U \subset U^{\prime}$ implies $U_{\beta, n} \subset U_{\beta, n}^{\prime}$, and $\bigcup_{n=1}^{\infty} U_{\beta, n}=U$. It remains to verify (a) and (b) for every $\alpha \in A$, and to check

(c) $U_{\beta, n}^{-} \subset U$. 
Now (a) is clear from the definitions. To prove (b) and (c), let

$$
U_{n}^{*}=\bigcup_{\alpha \in A}\left(U \cap C_{\alpha}\right)_{\alpha, n}^{-} .
$$

Clearly $U_{\beta, n} \subset U_{n}^{*} \subset U_{\beta, n}^{-}$. For each $\alpha \in A, U_{n}^{*} \cap C=\left(U \cap C_{\alpha}\right)_{\alpha, n}^{-}$, since $\left\{\left(\mathscr{C}_{\alpha}, S_{\alpha}\right)\right\}_{\alpha} \in_{A}$ is simply ordered. Hence $U_{n}^{*} \subset U$, and that implies both (b) and (c).

By Zorn's Lemma, let $\left(\mathscr{C}_{0}, S_{0}\right)$ be a maximal element of $G$. To complete the proof we need only show that $\mathscr{C}_{0}=\mathscr{B}$. Suppose not. Then there exists $E \in \mathscr{B}-\mathscr{C}_{0}$. Let $\mathscr{C}_{1}=\mathscr{C}_{0} \cup\{E\}$. Now $C_{0}$ and $E$ are closed stratifiable subspaces of $C_{1}=C_{0} \cup E$, and hence $C_{1}$ is stratifiable by the remark at the beginning of the proof. Thus, by Lemma 2.2 , one may obtain a stratification $S_{1}$ of $C_{1}$, say $U \rightarrow\left\{U_{1, n}\right\}$, such that

( a $) \quad U_{1, n} \cap C_{0}=\left(U \cap C_{0}\right)_{\alpha, n}$

(b') $U_{1, n}^{-} \cap C_{0}=\left(U \cap C_{0}\right)_{\alpha, n}^{-}$.

Consequently $\left(\mathscr{C}_{0}, S_{0}\right)<\left(\mathscr{C}_{1}, S_{1}\right)$, contradicting the maximality of $\left(\mathscr{C}_{0}, S_{0}\right)$. Hence $\mathscr{C}_{0}=\mathscr{B}$, and $X$ is stratifiable.

8. Perfect inverse images. It is well-known that the inverse image of a paracompact space, under a perfect map, is paracompact..$^{10}$ Clearly, the same cannot be said of metrizable spaces or of stratifiable spaces. However, we have the following (Upon completion of this work, we were informed that Okuyama [14] has an independent and quite different proof of the following Theorem 8.1. However, our proof is shorter and more elegant than his):

THEOREM 8.1. Suppose there exists a perfect map $f$ from the topological space $X$ to the metrizable space $Y$. Then $X$ is metrizable if and only if the diagonal in $X \times X$ is $a G_{\delta}$.

To prove the preceding theorem, we need the following lemma, which appears to be known, but we cannot find it in the literature.

Lemma 8.2. Let $X$ be a paracompact space with the diagonal a $G_{\delta}$ in $X \times X$. Then there exists a metrizable space $M$ and a continuous one-to-one map $j$ from $X$ onto $M$.

Proof. Let $\Delta$ be the diagonal of $X \times X$, and let $\Delta=\bigcap_{n=1}^{\infty} V_{n}$ such that each $V_{n}$ is open in $X \times X$.

By Theorem 28 and Lemma 30 (pp. 156-157) in [9], we can find

10 A perfect map $f: X \rightarrow Y$ is a closed continuous function, such that $f^{-1}(y)$ is compact for each $y \in Y$. 
a sequence $\left\{U_{n}\right\}_{n=1}^{\infty}$ of symmetric neighborhoods of $\Delta$ such that, for each $n$,

$$
U_{n+1} \circ U_{n+1} \circ U_{n+1} \subset U_{n} \cap V_{n} .
$$

By Theorem 12 (p. 185) in [9], letting $\mathscr{U}$ be the uniformity of $X$ for which $\left\{U_{n}\right\}_{n=1}^{\infty}$ is a base, $\left(X, \mathscr{C}_{6}\right)$ is pseudometrizable. However, since $\bigcap_{n=1}^{\infty} U_{n}=\Delta,(X, \mathscr{U})$ is $T_{1}$, and hence metrizable.

To complete the proof, denote $(X, \mathscr{C})$ by $M$ and let $j: X \rightarrow M$ be defined by $j(x)=x$, for each $x \in X$; the continuity of $j$ follows from Theorem 12 (b) on p. 185 in [9].

Proof of Theorem 8.1. The "if" assertion is obvious. Let us, therefore, prove the "only if" assertion.

By Lemma 8.2, there exists a metrizable space $M$ and a continuous one-to-one map $j: X \rightarrow M$. Now we define maps $h$ and $k$ such that

$$
h: X \rightarrow M \times Y, \quad k: M \times Y \rightarrow Y,
$$

with $k \circ h=f$, by letting

$$
h(x)=(j(x), f(x)), \quad x \in X,
$$

and

$$
k(m, y)=y, \quad m \in M, y \in Y .
$$

Clearly, $h$ and $k$ are continuous.

Hence, since $f$ is perfect, $h$ is a perfect map, by Proposition 5 (§10) in [3]. Therefore, since $h$ is one-to-one, $h$ is a homeomorphism into the metrizable space $M \times Y$. Consequently $X$ is metrizable.

The following is an immediate consequence of Theorem 8.1.

CoRollary 8.3. If $X$ is stratifiable and there exists a perfect map from $Y$ to a metrizable space, then $X$ is metrizable.

K. Morita [12] and Arhangel'skii [2] have extensively studied inverse images, under perfect maps, of metrizable spaces. By our Theorem 3.1, the corollary to Theorem 11 in [2], as well as Theorems 20 and 21 in [2], become immediate corollaries to our Theorem 8.1.

We now state the analogue of Theorem 8.1 for stratifiable spaces. 
THeOREM 8.4. Suppose there exists a perfect map $f$ from the topological space $X$ to the stratifiable space $Y$. Then $X$ is stratifiable if and only if the diagonal in $X \times X$ is $a G_{\delta}$.

Proof. The same as the proof of Theorem 8.1 since, by (A) and (B) in the introduction, stratifiability is productive and hereditary.

\section{BIBLIOGRAPHY}

1. R. Arens, Extension of functions on fully normal spaces, Pacific J. Math. 2 (1952), 11-22.

2. Arhangel'skii, On a class of spaces containing all metric and all locally bicompact spaces, Soviet Math (AMS Translations), 4 (1963), 1051-1055.

3. N. Bourbaki, Topologie Générale, Chapters I and II, Third Edition, Hermann, Paris, 1961.

4. J. G. Ceder, Some generalizations of metric spaces, Pacific J. Math. 11 (1961), 105126.

5. J. Dugundji, An extension of Tietze's theorem, Pacific J. Math. 1 (1951), 353-367.

6. Z. Frolik, On the topological product of paracompact spaces, Bull. Acad. Polonaise Science, 8 (1960), 747-750.

7. O. Hanner, Retraction and extension of mappings of metric and non-metric spaces, Ark. Mat. 2 (1952), 315-360.

8. S. T. Hu, Homotopy Theory, Academic Press, New York, 1959.

9. J. L. Kelley, General Topology, Van Nostrand, New York, 1955.

10. E. A. Michael, Some extension theorems for continuous functions, Pacific J. Math. 3 (1953), 789-806.

11. - Continuous Selection I, Ann. Math. 63 (1956), 361-382.

12. K. Morita, On the product of a normal space with a metric space, Proc. Japan Acad.

39 (1963), 148-150.

13. K. Morita and S. Hanai, Closed mappings and metric spaces, Proc. Japan Acad. 32 (1956), 10-14.

14. A. Okuyama, On metrizability of M-spaces, Proc. Japan Acad. 40 (1964), 176-179. 15. A. H. Stone, Metrizability of decomposition spaces, Proc. Amer. Math. Soc. 7 (1956), 690-700.

UNIVERSITY OF NEVADA 


\section{PACIFIC JOURNAL OF MATHEMATICS}

\section{EDITORS}

\section{H. SAMELSON}

Stanford University

Stanford, California

R. M. Blumenthal

University of Washington

Seattle, Washington 98105

\author{
*J. DugundJI \\ University of Southern California \\ Los Angeles, California 90007 \\ RICHARD ARENS \\ University of California \\ Los Angeles, California 90024
}

\section{ASSOCIATE EDITORS}
E. F. BECKENBACH
B. H. NeUManN
F. WolF
K. YosIDA

\section{SUPPORTING INSTITUTIONS}

UNIVERSITY OF BRITISH COLUMBIA
CALIFORNIA INSTITUTE OF TECHNOLOGY
UNIVERSITY OF CALIFORNIA
MONTANA STATE UNIVERSITY
UNIVERSITY OF NEVADA
NEW MEXICO STATE UNIVERSITY
OREGON STATE UNIVERSITY
UNIVERSITY OF OREGON
OSAKA UNIVERSITY
UNIVERSITY OF SOUTHERN CALIFORNIA

UNIVERSITY OF BRITISH COLUMBIA

UNIVERSITY OF CALIFORNIA

MONTANA STATE UNIVERSITY

NEW MEXICO STATE UNIVERSITY

OREGON STATE UNIVERSITY

OSAKA UNIVERSITY

UNIVERSITY OF SOUTHERN CALIFORNIA

\author{
STANFORD UNIVERSITY \\ UNIVERSITY OF TOKYO \\ UNIVERSITY OF UTAH \\ WASHINGTON STATE UNIVERSITY \\ UNIVERSITY OF WASHINGTON \\ AMERICAN MATHEMATICAL SOCIETY \\ CHEVRON RESEARCH CORPORATION \\ TRW SYSTEMS \\ NAVAL ORDNANCE TEST STATION
}




\section{Pacific Journal of Mathematics \\ Vol. 17, No. $1 \quad$ January, 1966}

Carlos Jorge Do Rego Borges, On stratifiable spaces ................ 1

Felix Earl Browder, Topological methods for non-linear elliptic equations of

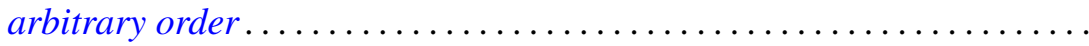

Gustave Choquet, Harry Corson and Victor Klee, Exposed points of convex

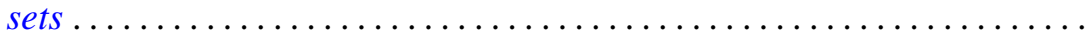

Phillip Emig, Remarks on the defect sum for a function meromorphic on an open Riemann surface ................................ 45

Ruth Goodman, A certain class of polynomials .................. 57

Sidney (Denny) L. Gulick, The bidual of a locally multiplicatively-convex

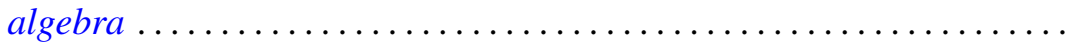

Eugene Carlyle Johnsen, Integral solutions to the incidence equation for finite projective plane cases of orders $n \equiv=2(\bmod 4) \ldots \ldots \ldots \ldots . .67$

Charles N. Kellogg, Centralizers and $H^{*}$-algebras .................. 121

Michael Lodato, On topologically induced generalized proximity relations. II .......................................... 131

P. H. Maserick, Half rings in linear spaces ..................... 137

Kathleen B O'Keefe, On a problem of J. F. Ritt .................... 149

Galen Lathrop Seever, Nonnegative projections on $C_{0}(X) \ldots \ldots \ldots \ldots$

Lawrence A. Shepp, Gaussian measures in function space ............ 167

Robert Charles Thompson, Classes of definite group matrices ........... 175 